\title{
PREVALÊNCIA DOS FATORES DE RISCO DA TUBERCULOSE EM IDOSOS NO ANO DE 2010 A 2019, NO ESTADO DE SÃO PAULO
}

\author{
PREVALENCE OF RISK FACTORS FOR TUBERCULOSIS IN THE ELDERLY \\ IN THE YEAR 2010 TO 2019, IN THE STATE OF SÃO PAULO
}

\begin{abstract}
Tainara Págio Chagas ${ }^{1 \star}$, Paloma Lima Borôto ${ }^{1}$, lago Esquerdo Norte ${ }^{1}$, Letícia Gomes Alves².

${ }^{1}$ Universidade Vila Velha, Vila Velha, ES, Brasil.

${ }^{2}$ Instituto Tocantinense Presidente Antônio Carlos, Porto Nacional, TO, Brasil.

*tainarapagioc@hotmail.com
\end{abstract}

\section{RESUMO}

A tuberculose é uma doença infecciosa transmitida predominantemente por via aérea, de evolução crônica, que compromete principalmente os pulmões, cujo agente etiológico é a bactéria Mycobacterium tuberculosis (MT). Apesar de já existirem recursos tecnológicos para seu tratamento, permanece na atualidade como um importante problema de saúde pública mundial, em virtude da ampla dispersão geográfica e a emergência de casos multirresistentes. Destacam-se como fatores de risco da tuberculose em idosos: diabetes, tabagismo, alcoolismo, AIDS, doenças mentais e drogas ilícitas. O trabalho tem como propósito verificar a prevalência dos fatores de risco para tuberculose em idosos a partir de 65 anos de idade em São Paulo. Foi realizado um estudo transversal descritivo epidemiológico, sobre a prevalência da tuberculose e dos seus principais fatores de risco em idosos, por meio do Portal do Ministério da Saúde, DATASUS, através de dados secundários do Sistema de Informação de Agravos e Notificação (SINAN). Os critérios de inclusão avaliados foram os fatores de risco, a faixa etária e o período de notificação. Foram observados 13548 casos de tuberculose em idosos no período avaliado. A maior prevalência esteve no ano de 2019, com 1623 doentes. O ano de 2010 marcou a menor prevalência, com 1236 casos. De acordo com os dados, dentro dos fatores de risco, houve uma prevalência de diabetes com 2400 casos, seguido do tabagismo com 1823, alcoolismo 1353, AIDS 273, doenças mentais 255 e drogas ilícitas 111. A frequência relativa do fator de risco mais predominante é de $17,71 \%$ de diabetes, enquanto a menor é de $0,82 \%$ referente a drogas ilícitas. Visto que, a frequência relativa dos fatores como o tabagismo, alcoolismo, AIDS e doenças mentais, constam respectivamente com $13,45 \%$, $9,99 \%, 2,0 \%$ e $1,88 \%$. Portanto, observou-se que os casos de tuberculose aumentaram significativamente em idosos no Estado de São Paulo. Além disso, a diabetes foi o fator de risco mais associado com a tuberculose, representando $17,71 \%$ dos casos notificados. Dessa forma, é necessário investir em políticas públicas destinadas a prevenção da tuberculose e dos fatores de risco para essa doença, principalmente a diabetes que obteve maior impacto nos casos avaliados. Vale ressaltar que apesar da imunização ativa para a tuberculose ser obrigatória ao recém-nascido, medidas de incentivo a prevenção dos fatores de risco como a alimentação saúdavel, a prática de exercícios físicos, uso de preservativo e o não uso drogas lícitas e ilícitas, 
devem ser tomadas.

Palavras-chave: Epidemiologia. Fatores de risco. Idosos. Prevalência. Tuberculose. 